\title{
Compression screw fixation without bone grafting for scaphoid fibrous nonunion
}

\author{
Jeremy S. Somerson • Daniel J. Fletcher • \\ Ramesh C. Srinivasan • David P. Green
}

Published online: 18 February 2015

(C) American Association for Hand Surgery 2015

\begin{abstract}
Purpose Bone graft is often recommended as an adjuvant for treatment of scaphoid nonunions. However, recent literature has suggested that fibrous nonunion may be suited to treatment with rigid fixation without bone grafting. This work reported on outcomes of compression screw fixation for established scaphoid fibrous nonunions without bone graft. Methods Fourteen patients underwent surgical compression screw fixation without bone grafting of scaphoid fibrous nonunion between January 1, 2000, and December 31, 2012, with minimum follow-up until the time of clinical and radiographic healing. Fibrous nonunion of the scaphoid was defined as a scaphoid fracture with all of the following features: (1) persistent tenderness, (2) incomplete trabecular bridging on three Xray views, (3) injury that had occurred at a minimum of 6 months prior to surgery, and (4) identification of fibrous union at the time of surgery. Outcomes were assessed with range of motion assessment, Disability of the Arm, Shoulder and Hand (DASH) scores, and plain radiographs.

Results Twelve of the 14 patients healed successfully, while two patients required secondary vascularized bone grafting. Both unhealed patients sustained proximal pole fractures and had a duration of $\geq 1$ year from injury to surgery. Average time to healing was $4.4 \pm 2.0$ months. Average flexion was $73 \pm 22^{\circ}$ and average extension was $66 \pm 22^{\circ}$ postoperatively. Average grip strength was $90 \pm 25 \mathrm{lbs}$ on the operative side. Mean postoperative pain score was 1.4 (range, 0 to 7 ). Mean postoperative DASH score was 10.2 (range, 0 to 52). Increasing age
\end{abstract}

D. J. Fletcher · R. C. Srinivasan • D. P. Green

The Hand Center of San Antonio, 21 Spurs Lane, San

Antonio, TX 78240, USA

J. S. Somerson $(\bowtie)$

Department of Orthopaedics, University of Texas Health Science

Center San Antonio, San Antonio, TX, USA

e-mail: jeremysomerson@gmail.com and an interval from injury to surgery of $>1$ year correlated with worse DASH and pain scores.

Conclusions Patients with fibrous scaphoid nonunion demonstrated good results with rigid fixation without bone grafting. Increasing age and $>1$-year interval between injury and surgery resulted in lower self-assessed outcomes.

Level of Evidence Therapeutic Level IV, retrospective case series

Keywords Scaphoid · Nonunion · Compression screw

\section{Introduction}

Scaphoid nonunion represents a debilitating condition that frequently leads to wrist malalignment and arthritis if untreated $[9,11,12,14,19]$. A wide range of surgical treatment options have been reported, from percutaneous fixation to microsurgical vascularized graft techniques. [2] Recommendations for surgical options vary depending on the timeframe from injury, previous surgery, and amount of displacement.

Fibrous nonunion of the scaphoid was described in detail by Barton in a small series of patients with osseous nonunion and an overlying stable fibrocartilage surface; half of these cases proceeded to persistent nonunion [1]. Treatment recommendations for fibrous scaphoid nonunions are remarkably uniform among primary orthopedic and hand surgery textbooks: all referenced sources encourage rigid fixation with the addition of bone grafting $[5,7,8,17]$.

Weber and Cech described two basic types of nonunions in long bones - vital and avital-depending on the underlying biological healing capacity; vital fractures were proposed to lack only mechanical stability [21]. A number of authors have reported success in small series of scaphoid nonunions treated 
with stabilization without bone grafting, although inclusion criteria and outcome assessment measures vary within these publications $[3,4,6,10,13,15,16,18]$. In particular, definitions of "fibrous" nonunions were defined heterogeneously based on static imaging or intraoperative exploration. Unlike prior publications, this work used a minimum 6-month time from injury and did not require prior arthroscopic evaluation. Prognostic factors were evaluated for associations with final outcome and healing rate.

In symptomatic patients with absent interfragmentary motion, internal fixation was performed without bone grafting. The purpose of this work is to report the results of open compression screw fixation without bone grafting in symptomatic fibrous nonunions of the scaphoid.

\section{Materials and Methods}

Between December 7, 2000, and February 28, 2014, 17 consecutive patients meeting the inclusion criteria were treated for scaphoid fibrous nonunion by the senior authors using compression screw fixation without bone grafting. All patients underwent initial conservative treatment with casting. Fourteen of these patients were followed until the time of radiographic and clinical healing, and three were lost to follow-up. The risks, benefits, and alternatives to surgery were discussed in detail, and all patients provided informed written consent. Approval from the institutional review board (IRB) was obtained, and a retrospective chart review was undertaken. All procedures followed were in accordance with the ethical standards of the responsible committee on human experimentation (institutional and national) and with the Helsinki Declaration of 1975 , as revised in 2008 .

The criteria outlined in the Strengthening the Reporting of Outcome Studies in Epidemiology (STROBE) statement [20] were followed when applicable to a case series. Collected data included age, timepoint of radiographic and clinical healing, flexion/extension arc at postoperative visits, grip strength, visual analog scale (VAS) pain scores, Disability of the Arm, Shoulder and Hand (DASH) scores, return to previous activity level, and complications. Computerized tomography was not routinely used due to cost and risks of ionizing radiation.

\section{Inclusion/Exclusion Criteria}

Patients were included if they met the following criteria: (1) persistent tenderness, (2) incomplete trabecular bridging on three X-ray views, (3) injury that had occurred at a minimum of 6 months prior to surgery, and (4) identification of fibrous union at the time of surgery. Patients were excluded if they exhibited carpal collapse, avascular necrosis, significant displacement, or mid-carpal instability. Fibrous nonunion was confirmed if a patient meeting the above criteria was found to have no radiographic or intraoperative evidence of motion at the fracture site. This was evaluated with an ulnar-deviated posteroanterior stress X-ray that demonstrated no increased displacement compared to a standard PA radiograph (Fig. 1) and/or intraoperative inspection after an open approach. Patients were excluded if they had undergone previous surgery.

\section{Data Collection}

Preoperative and latest postoperative data were obtained from clinic notes. Flexion/extension arc was measured using a hand-held goniometer. Grip strength was measured using a hand-held dynamometer. Patient-reported VAS pain score and DASH score were collected and recorded into notes.

\section{Surgical Technique and Postoperative Course}

All surgeries were performed by the senior authors (DPG, RCS) using an open approach. Proximal third fractures were treated with a dorsal approach, and middle third fractures were treated with a volar or dorsal approach. A guide wire was placed from the proximal pole of the scaphoid into the central axis as confirmed by fluoroscopy. A headless compression screw (Acutrak; Acumed: Hillsboro, OR) was placed over the guidewire after measuring appropriate length. A median screw length of $20 \mathrm{~mm}$ was used (range, 16 to $25 \mathrm{~mm}$ ). No bone grafting or biological adjuvants were used during surgery.

Postoperatively, a short arm splint was applied. Two patients were immobilized for 2 weeks and started on early active and passive motion exercises with a splint for comfort. The remaining patients were immobilized for an average of 10.8 weeks (range, 8.0 to 13.0 ). Patients underwent a progressive course of physician-directed hand therapy to improve range of motion and strength. Regular follow-ups were performed at 2, 6, and 12 weeks after surgery. Subsequent followup visits continued until symptoms resolved. Union was verified clinically by resolution of pain with palpation and radiographically with the appearance of complete bridging trabeculae on three X-ray views.
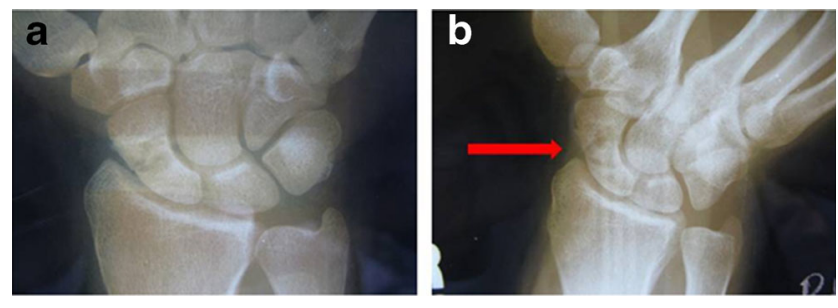

Fig. 1 Fibrous scaphoid nonunion without increased gapping or displacement on the posteroanterior (PA) ulnar deviated view (right) when compared to the standard PA view (left) 


\section{Statistical Analysis}

Descriptive statistics were calculated and reported in the format mean \pm standard deviation (SD) or with ranges where appropriate. Univariate modeling of prognostic factors between groups was performed using a nonparametric Wilcoxon rank sum test chi-square approximation for nominal variables and a bivariate linear logistic regression with analysis of variance for continuous variables. Significance was set at $p \leq 0.05$.

\section{Results}

Mean age was $21.1 \pm 10.6$ years old. Thirteen patients were male $(93 \%)$ and one was female. There were nine fractures of the proximal third of the scaphoid and five middle third fractures. The mean time from injury to surgery was 13.6 months (range, 7.0 to 24.1 months). Twelve of the 14 patients healed at an average of $4.4 \pm 2.0$ months (range, 2.0 to 8.0 months). Two patients did not heal and underwent vascularized bone grafting; these two patients remained moderately symptomatic at latest follow-up with DASH scores of 24 and 33. Average flexion was $73 \pm 22$ degrees and average extension was $66 \pm 22^{\circ}$ postoperatively. Average grip strength was $90 \pm 25 \mathrm{lbs}$ on the operative side and $98 \pm 27 \mathrm{lbs}$ on the nonoperative side at latest follow-up. Mean postoperative pain score was 1.4 (range, 0 to 7 ). Mean postoperative DASH score was 10.2 (range, 0 to 52). Eleven out of 12 patients that healed returned to their previous level of activity, reporting no symptoms or only mild discomfort with heavy lifting, while one patient reported persistent pain and need for activity modifications.

\section{Prognostic Factors}

Univariate analysis was performed for age and fracture location using pain and DASH scores at latest follow-up as endpoints. Increasing age correlated with higher DASH $\left(R^{2}=0.6733 ; p=0.0006\right)$ and pain score $\left(R^{2}=\right.$ $0.6131 ; p=0.0009)$. Patients who underwent surgery $<1$ year after the date of injury $(n=7)$ uniformly reported pain and DASH scores of 0 , which was significantly lower than patients who underwent surgery $>1$ year after injury ( $n=7$; pain $2.9 \pm 2.5, p=0.01$; DASH $22.0 \pm 20.0$, $p=0.02$ ). Patients with fractures to the proximal third of the scaphoid had a trend toward higher DASH (mean, 16.5; range, 0 to 52) and pain scores (mean, 2.2; range, 0 to 7 ) compared to those with fractures of the middle third, all of whom reported no pain and a DASH score of 0 (DASH 0.0, $p=0.09$; pain score $0.0, p=0.06$ ).
Complications and Revisions

There were three complications: one patient developed superficial cellulitis that was successfully treated with oral antibiotic treatment and two patients required vascularized bone grafting for persistent nonunion. Both patients requiring a second procedure had fractures of the proximal pole and had been injured $>1$ year prior to surgery.

\section{Discussion}

This study was performed to evaluate the results of compression screw fixation without bone grafting in symptomatic fibrous nonunions of the scaphoid. Prognostic factors including age and fracture location were evaluated for effects on outcomes at latest follow-up. The majority ( $86 \%$ ) of patients healed without requiring further surgery, while two patients with proximal pole injuries and duration of nonunion $>1$ year from the time of injury underwent subsequent vascularized bone grafting. Results were better in younger patients and patients with a shorter time interval from injury to surgery.

Cosio et al. reported results of multiple percutaneous pinning of symptomatic scaphoid nonunions without bone grafting [6]. Inclusion criteria were clinically symptomatic nonunions confirmed by radiographs that interfered with activities of work or daily living. All patients were at least 4 months post-injury. Eighteen patients underwent pinning with two to four 0.045-in. wires and immobilized until healed. Follow-up data was available for 13 patients (72\%) and 10 of $13(77 \%)$ had healed successfully. Although that study had limited clinical follow-up, it demonstrated the potential of scaphoid nonunions to heal without addition of bone graft, similar to the results reported in this work.

A series of 50 scaphoid nonunions treated with Herbert screw fixation and an analysis of prognostic factors were reported by Shah and Jones [16]. Avascular necrosis and prior nonunion surgery were found to be predictive of poor outcome. Of note, the authors found that failure to bone graft in selected patients with an "intact cartilaginous envelope or a stable firm fibrous union" did not negatively affect results. In contrast to the present study, these authors included several patients with long-term nonunions greater than 5 years postinjury. Among the eight fractures considered to be a "firm fibrous union," five were at the waist and three were at the proximal pole. All eight healed with bony union and excellent or good results.

Slade et al. reported results from a consecutive series of 15 scaphoid fibrous unions treated with percutaneous screw fixation without bone grafting [18]. The presence of fibrous union was determined by arthroscopic evaluation. At an average of 14 weeks, all patients healed as confirmed by examination, radiographs, and computerized tomography (CT). 
More recently, Mahmoud and Koptan reported results from a consecutive series of 27 scaphoid nonunions treated with percutaneous screw fixation without bone grafting [13]. Fracture union was confirmed in all patients at a mean of 11.6 weeks using clinical examination, radiographs, and CT. Patients were excluded if they had humpback deformity or arthritis, but all patients had extensive local resorption of bone. In contrast to the present study, all patients underwent preoperative CT scan to evaluate remaining bone stock and quality.

Limitations of this work include those inherent to a retrospective series, including the potential for selection and reporting bias. Further, the results are those of a tertiary referral center at an academic practice, which may not be applicable to all practice environments. Finally, the relatively small size of the patient cohort limits the generalizability of the results.

This series suggest that compression screw fixation without bone graft may be an option for scaphoid fibrous nonunion with carefully selected patients. Clinical and radiographic healing was observed in 12 of 14 patients (86\%). Both unhealed fractures were of the proximal pole in patients with a duration of $>1$ year from injury to surgery. Fluoroscopic and intraoperative evaluation can adequately guide treatment and determine whether interfragmentary motion is present. Further study is needed to assess prognostic factors and to establish non-inferiority compared to the current gold standard treatment.

Conflict of Interest Jeremy S. Somerson declares that he has no conflict of interest.

Daniel J. Fletcher declares that he has no conflict of interest.

Ramesh C. Srinivasan declares that he has no conflict of interest.

David P. Green declares that he has no conflict of interest.

Statement of Human and Animal Rights All procedures followed were in accordance with the ethical standards of the responsible committee on human experimentation (institutional and national) and with the Helsinki Declaration of 1975, as revised in 2008.

Statement of Informed Consent Informed consent was obtained from all patients for being included in the study.

\section{References}

1. Barton NJ. Apparent and partial non-union of the scaphoid. J Hand Surg Br Eur Vol. 1996;21(4):496-500. doi:10.1016/S0266-7681(96) 80054-4.

2. Buijze GA, Ochtman L, Ring D. Management of scaphoid nonunion. J Hand Surg. 2012;37(5):1095-100. doi:10.1016/j.jhsa.2012.03.002.
3. Bumbaširević $M$, Tomić $S$, Lešić $A$, Bumbaširević $V$, Rakočević $Z$, Atkinson HD. The treatment of scaphoid nonunion using the Ilizarov fixator without bone graft, a study of 18 cases. J Orthop Surg. 2011;6: 57. doi:10.1186/1749-799X-6-57.

4. Capo JT, Shamian B, Rizzo M. Percutaneous screw fixation without bone grafting of scaphoid non-union. Isr Med Assoc J IMAJ. 2012;14(12):729-32.

5. Cassidy C, Ruby L. Fractures and dislocations of the carpus. In: Browner BD, Jupiter JB, Levine AM, Trafton PG, Krettek C, Editors. Skeletal trauma. 4th ed. Saunders; 2009:1360.

6. Cosio MQ, Camp RA. Percutaneous pinning of symptomatic scaphoid nonunions. J Hand Surg. 1986;11(3):350-5.

7. Gaebler C, McQueen M. Carpus fractures and dislocations. In: Bucholz R, Heckman JD, Court-Brown CM, Tornetta III P, editors. Rockwood and Green's fractures in adults. 7th ed. Philadelphia: Williams and Wilkins; 2010. p. 800.

8. Geissler W, Slade J. Fractures of the carpal bones. In: Wolfe SW, Hotchkiss RN, Pederson WC, Kozin SH, editors. Green's operative hand surgery. 6th ed. Philadelphia: Elsevier; 2011. p. 662.

9. Inoue G, Sakuma M. The natural history of scaphoid non-union. Radiographical and clinical analysis in 102 cases. Arch Orthop Trauma Surg. 1996;115(1):1-4.

10. Ledoux P, Chahidi N, Moermans JP, Kinnen L. Percutaneous Herbert screw osteosynthesis of the scaphoid bone. Acta Orthop Belg. 1995;61(1):43-7.

11. Lindström G, Nyström Å. Natural history of scaphoid non-union, with special reference to "asymptomatic" cases. J Hand Surg Br. 1992;17(6):697-700. doi:10.1016/0266-7681(92)90204-F.

12. Mack GR, Bosse MJ, Gelberman RH, Yu E. The natural history of scaphoid non-union. J Bone Joint Surg Am. 1984;66(4):504-9.

13. Mahmoud M, Koptan W. Percutaneous screw fixation without bone grafting for established scaphoid nonunion with substantial bone loss. J Bone Joint Surg (Br). 2011;93-B(7):932-6. doi:10.1302/0301620X.93B7.25418.

14. Ruby LK, Stinson J, Belsky MR. The natural history of scaphoid non-union. A review of fifty-five cases. J Bone Joint Surg Am. 1985;67(3):428-32.

15. Saint-Cyr M, Oni G, Wong C, Sen MK, LaJoie AS, Gupta A. Dorsal percutaneous cannulated screw fixation for delayed union and nonunion of the saphoid. Plast Reconstr Surg. 2011;128(2):467-73. doi: 10.1097/PRS.0b013e31821e703b.

16. Shah J, Jones WA. Factors affecting the outcome in 50 cases of scaphoid nonunion treated with Herbert screw fixation. J Hand Surg Br. 1998;23(5):680-5.

17. Slade J, Merrell G. Percutaneous treatment of grade I to III scaphoid nonunions. In: Wiesel SW, editor. Operative techniques in orthopaedic surgery. Philadelphia: Lippincott, Williams and Wilkins; 2011. p. 2260.

18. SladeIII JF, Geissler WB, Gutow AP, Merrell GA. Percutaneous internal fixation of selected scaphoid nonunions with an arthroscopically assisted dorsal approach. J Bone Joint Surg. 2003;85(suppl_4):20-32.

19. Vender MI, Watson HK, Wiener BD, Black DM. Degenerative change in symptomatic scaphoid nonunion. J Hand Surg. 1987;12(4):514-9.

20. Von Elm E, Altman DG, Egger M, et al. The Strengthening the Reporting of Observational Studies in Epidemiology (STROBE) statement: guidelines for reporting observational studies. Lancet. 2007;370(9596):1453-7. doi:10.1016/S0140-6736(07)61602-X.

21. Weber B, Cech O. Pseudarthrosis. Bern: Hans Huber; 1976. 